\title{
New options with dabigatran etexilate in anticoagulant therapy
}

\author{
This article was published in the following Dove Press journal: \\ Vascular Health and Risk Management \\ 8 May 2010 \\ Number of times this article has been viewed
}

\section{Lars Maegdefessel \\ Joshua M Spin \\ Junya Azuma \\ Philip S Tsao}

Department of Cardiovascular Medicine, Stanford University School of Medicine, Stanford, CA, USA

Correspondence: Lars Maegdefessel Stanford University School of Medicine, Division of Cardiovascular Medicine, 300 Pasteur Drive, Stanford,

CA 94305-5406, USA

Tel +I $650724-5075$

Fax + I 650 725-I599

Email maegdefessel@stanford.edu
Abstract: Thrombosis, the localized clotting of blood, occurs in both the arterial and venous circulation, and has a major impact on health outcomes. The primary etiology of myocardial infarctions, and approximately $80 \%$ of strokes, is acute arterial thrombosis. In combination this represents the most common cause of death in the Western world, while the third leading cause of cardiovascular-associated death is venous thromboembolism. An understanding of the pathogenic changes in the vessel wall and the blood that result in thrombosis is crucial for developing safer and more effective antithrombotic drugs. Dabigatran etexilate belongs to a new class of direct thrombin inhibitors. Following oral administration, dabigatran reaches peak plasma concentrations within 2 hours, shows linear pharmacokinetics, and a limited (but important) amount of direct drug interactions. Given once daily at $150 \mathrm{mg}$ or $220 \mathrm{mg}$, it has proven to be competitive with enoxaparin in the prevention of venous thromboembolism after major orthopedic surgery, with a comparable safety profile. For stroke prevention in patients suffering from atrial fibrillation, dabigatran administered at a dose of $110 \mathrm{mg}$ twice daily was associated with rates of stroke and systemic embolism that were similar to those associated with warfarin, as well as lower rates of hemorrhage. Dabigatran given at a dose of $150 \mathrm{mg}$ twice daily, as compared with warfarin, was associated with lower rates of stroke and systemic embolism but similar rates of major hemorrhage. Oral bioavailability of dabigatran, together with a rapid onset and offset of action and predictable anticoagulation response, makes this newly available antithrombotic drug an attractive alternative to traditional anticoagulant therapies for numerous thrombosis-related indications.

Keywords: dabigatran etexilate, anticoagulation, direct thrombin inhibitors, thrombosis, prevention

\section{Indications for anticoagulant therapy}

Thrombosis, the formation of a blood clot within a blood vessel or cavity of the heart, is a leading cause of death and disability. Up to $15 \%$ of patients hospitalized for an acute medical illness develop venous thromboembolic disease and some of them suffer from serious and life-threatening complications such as pulmonary embolism. ${ }^{1}$ Thromboemboli are blood clots moving through the bloodstream, which may lodge in a vessel and block circulation. The following paragraph summarizes the major indications for anticoagulant therapy and address specific complications and common limitations of current therapeutic regimens.

\section{Venous thromboembolic disease}

Although many associate venous thrombosis with recent trauma or surgery, $50 \%$ to $70 \%$ of symptomatic cases, as well as the majority of cases of fatal pulmonary embolism (PE), submit your manuscript | www.dovepress.com

Dovepress

8942 
occur in medical patients. ${ }^{2}$ Due to the often silent nature of venous thrombosis, the first sign of a problem may be a clinically significant complication, such as PE. Cancer and other medical illness are major contributors to venous thromboembolism (VTE) risk. ${ }^{3}$ Hospitalized patients, especially the ones in critical care settings, are often immobile or even bedridden. Even in the absence of medical illness, lack of mobility can lead to venous stasis and VTE, as can occur during long-distance air travel. ${ }^{4}$ Table 1 presents common conditions and patientrelated, predisposing characteristics that are associated with hospitalization and an increased risk for VTE. Age, in particular, is one of the most important risk factors for VTE. ${ }^{1}$

Prospective studies have shown that hospitalized medical patients at high risk who do not receive preventive anticoagulant therapy develop isolated calf thrombosis in $10 \%$ to $15 \%$ of cases. The same studies revealed an incidence of proximal deep vein thrombosis (DVT) in $2 \%$ to $5 \%$ and of $\mathrm{PE}$ in $0.3 \%$ to $1.5 \%$. $^{5,6}$

Studies have also shown that anticoagulant prophylaxis reduces the risk of symptomatic VTE in hospitalized medical patients. ${ }^{7}$ Despite the clear need for prophylactic care to prevent VTE in high-risk medical patients, a recent multinational, cross-sectional report ${ }^{3}$ revealed that fewer than $40 \%$ of hospitalized medical patients at risk received standard VTE prophylaxis. Therefore, timely risk assessment and preventive therapy is crucial.

In addition to the acute risk of $\mathrm{PE}$, a potentially lifethreatening event, venous thrombosis also poses risks of

Table I Common conditions and patient-related predisposing characteristics associated with hospitalization and increased risk for VTE

\begin{tabular}{|c|c|}
\hline Conditions associated & previous stroke \\
\hline with hospitalization that & congestive heart failure (NYHA Class III-IV) \\
\hline \multirow[t]{5}{*}{ increase risk of VTE } & acute respiratory disease \\
\hline & acute myocardial infarction \\
\hline & acute arthritis \\
\hline & acute infection \\
\hline & inflammatory bowel disease \\
\hline Patient-related & recent surgery or major trauma \\
\hline predisposing & immobility or paralysis \\
\hline characteristics that & malignancy \\
\hline \multirow[t]{8}{*}{ increase risk of VTE } & previous VTE \\
\hline & older age ( $>80$ years) \\
\hline & estrogen therapy (contraceptives or \\
\hline & hormone replacement) \\
\hline & obesity \\
\hline & central vein catheterization \\
\hline & varicose veins \\
\hline & inherited or acquired thrombophilia. \\
\hline
\end{tabular}

Abbreviations: VTE, venous thromboembolism; NYHA, New York Heart Association (classification). intermediate- and long-term complications that include recurrent DVT, post-thrombotic syndrome, and chronic thromboembolic pulmonary hypertension.

\section{Recurrent DVT}

The risk of recurrent DVT varies depending on the initial treatment. With no treatment, about half of the patients with symptomatic proximal DVT or PE experience a recurrence within 3 months. ${ }^{8}$ When proximal DVT is treated with at least 3 months of effective anticoagulation, the recurrence rate decreases considerably to $4 \%$. Further duration of treatment is strongly dependant on the etiology of the thrombosis (idiopathic vs secondary). Indefinite anticoagulation might be required for unexplained cases of recurrent DVT. ${ }^{9}$

\section{Post-thrombotic syndrome}

Between $20 \%$ and $50 \%$ of those who develop DVT eventually develop a condition known as post-thrombotic syndrome (PTS). ${ }^{10}$ The exact cause of PTS is not well understood, but it may involve damage to venous valves, ultimately leading to increased intravenous pressure. ${ }^{11}$ The main symptom is chronic pain. Signs of PTS include swelling, discoloration of the affected leg, and in severe cases, skin ulceration. PTS is not reliably prevented by prompt antithrombotic therapy following the diagnosis of DVT. This is another reason why preventive therapy is the optimal approach for high-risk patients. ${ }^{12}$

\section{Chronic thromboembolic pulmonary hypertension}

Pulmonary hypertension occurs as a late complication in between $3 \%$ and $4 \%$ of patients who survive pulmonary embolism. ${ }^{13}$ Symptoms include progressive shortness of breath and exercise intolerance. Later in the course of the disorder, chest pain with exertion and syncope may occur. Without intervention, the 5-year survival rate once the mean pulmonary artery pressure has reached $40 \mathrm{mmHg}$ is about $30 \%{ }^{14}$

\section{Atrial fibrillation and stroke prevention}

Atrial fibrillation (AF) is the most common sustained arrhythmia seen in clinical practice, affecting an estimated 4.5 million people in the European Union and 2.2 million Americans. ${ }^{15} \mathrm{AF}$ is associated with a major risk of stroke, caused by thrombus formation within the left atrium and embolization to the cerebral arteries. The degree of stroke risk and the need for anticoagulant therapy to lower this risk varies among patients with AF.

The prevalence of this arrhythmia increases sharply with older age. AF is uncommon among individuals $\leq 50$ years old. ${ }^{16}$ In the Framingham Heart Study, the percentage risk 
of stroke ascribable to AF rose from $1.5 \%$ in the age group 50 to 59 years, to $23.5 \%$ in the age group 80 to 89 years. The median age of patients with AF is 72 years. ${ }^{17}$ Worldwide population trends of increasing life expectancy and increasing prevalence of selected risk factors for AF indicate a worsening epidemic. ${ }^{18}$ Overall, AF accounts for about $15 \%$ of all strokes in the United States. ${ }^{16}$ The rate of ischemic stroke among AF patients included in clinical primary prevention trials and not treated with antithrombotic therapy averaged $4.5 \%$ per year, similar to estimates of stroke risk from the Framingham Heart Study. Further analyses from these studies indicate that the lifetime risk of AF for an individual age 40 years is about $25 \% .{ }^{19} \mathrm{AF}$ is more prevalent in men than in women at all ages. The prevalence of AF may actually be even higher, given the potential for AF to remain undiagnosed. ${ }^{20}$

$\mathrm{AF}$ raises the risk of ischemic stroke by a factor of four to five, thought to be predominately the result of cardiogenic thomboembolism. ${ }^{21}$ This is based on clinical assessments, on operative findings of intracardiac thrombus in patients with rheumatic mitral valve disease, and more recently, on transesophageal echocardiography imaging of thrombus in the left atrium of patients with AF, mainly in the left atrial appendage. ${ }^{16}$

Given the epidemiologic characteristics of AF and the importance of stroke as a leading cause of death and disability, multiple large-scale, randomized trials have been completed and are under way to find efficacious and relatively safe ways to reduce the risk of ischemic stroke and other systemic thromboembolic events related to AF.

\section{Acute coronary syndrome}

Acute coronary syndrome (ACS) occurs when an atherosclerotic plaque ruptures, leading to thrombus formation within a coronary artery. Patients who develop symptoms consistent with ACS, such as chest pain and diaphoresis, require timely evaluation to determine the cause. When ACS is diagnosed, further stratification into categories of STelevation myocardial infarction (STEMI), non-ST-elevation myocardial infarction (NSTEMI), and unstable angina guides therapeutic decision-making. ${ }^{22}$ Following recovery from an episode of ACS, patients continue to be at heightened risk of heart attack and stroke, for which a range of secondary preventive treatments are available. ${ }^{23}$

\section{Mechanical heart valve recipients}

Optimal anticoagulation management in patients after mechanical heart valve replacement is still an unresolved issue. Vitamin K antagonists (VKAs) represent the standard of care for long-term prophylaxis after mechanical heart valve replacement. Nevertheless, thromboembolic events occur in approximately $2 \%$ to $5 \%$ of these patients per year despite oral anticoagulation (OAC). ${ }^{24}$ The incidence of bleeding complications correlates with VKA dosage, and such complications have been observed within the range of $2 \%$ to $9 \%$ annually. ${ }^{25}$ Thromboembolism and anticoagulant-related bleeding account for $\approx 75 \%$ of all complications experienced by heart valve recipients. ${ }^{26}$ They occur in particular in patients with mechanical heart valves in need of invasive or surgical procedures when the antithrombotic therapy is switched from VKAs to heparins or vice versa. ${ }^{26,27}$ Therefore, it is important to find alternative approaches for the prophylaxis of thromboembolic complications after mechanical heart valve replacement that are associated with fewer bleeding events.

\section{Benefits and limitations of current anticoagulation regimen}

While most current anticoagulant drugs (Figure 1) have been mainstays of antithrombotic therapy for decades, many clinicians find these medications challenging to use. This, when combined with underappreciation of the degree of VTE risk in certain patients, has led to considerable underuse of preventive antithrombotic therapy in some countries. Observational studies reveal that fewer than half of the patients at high risk of VTE receive prophylactic treatment. ${ }^{2,5}$

A new anticoagulant that more closely meets the criteria for optimal antithrombotic efficacy could improve the quality of care. Such an advance also might help overcome the gaps between evidence-based treatment recommendations and clinical practice. Ideally, an anticoagulant should be administered orally. It should be highly effective in reducing venous thromboembolism, inhibiting both free and clot-bound coagulation factors, have a predictable dose response and kinetics, and show low, nonspecific plasma protein binding, resulting in a low rate of bleeding events. Coagulation monitoring and dose adjustment should not be routinely required, thus creating a wide therapeutic window. Furthermore, there should be only little interaction with food or other drugs.

\section{Vitamin $\mathrm{K}$ antagonists}

Antagonists of vitamin $\mathrm{K}$ have been used as anticoagulants for over 50 years. Warfarin, a synthetic derivative of coumarin, is the most commonly used VKA in the US. In some European countries, other coumarin derivatives (phenprocoumon and acenocoumarol) are used instead. ${ }^{28}$

With appropriate dosing, these medications effectively inhibit coagulation and have been shown to substantially 


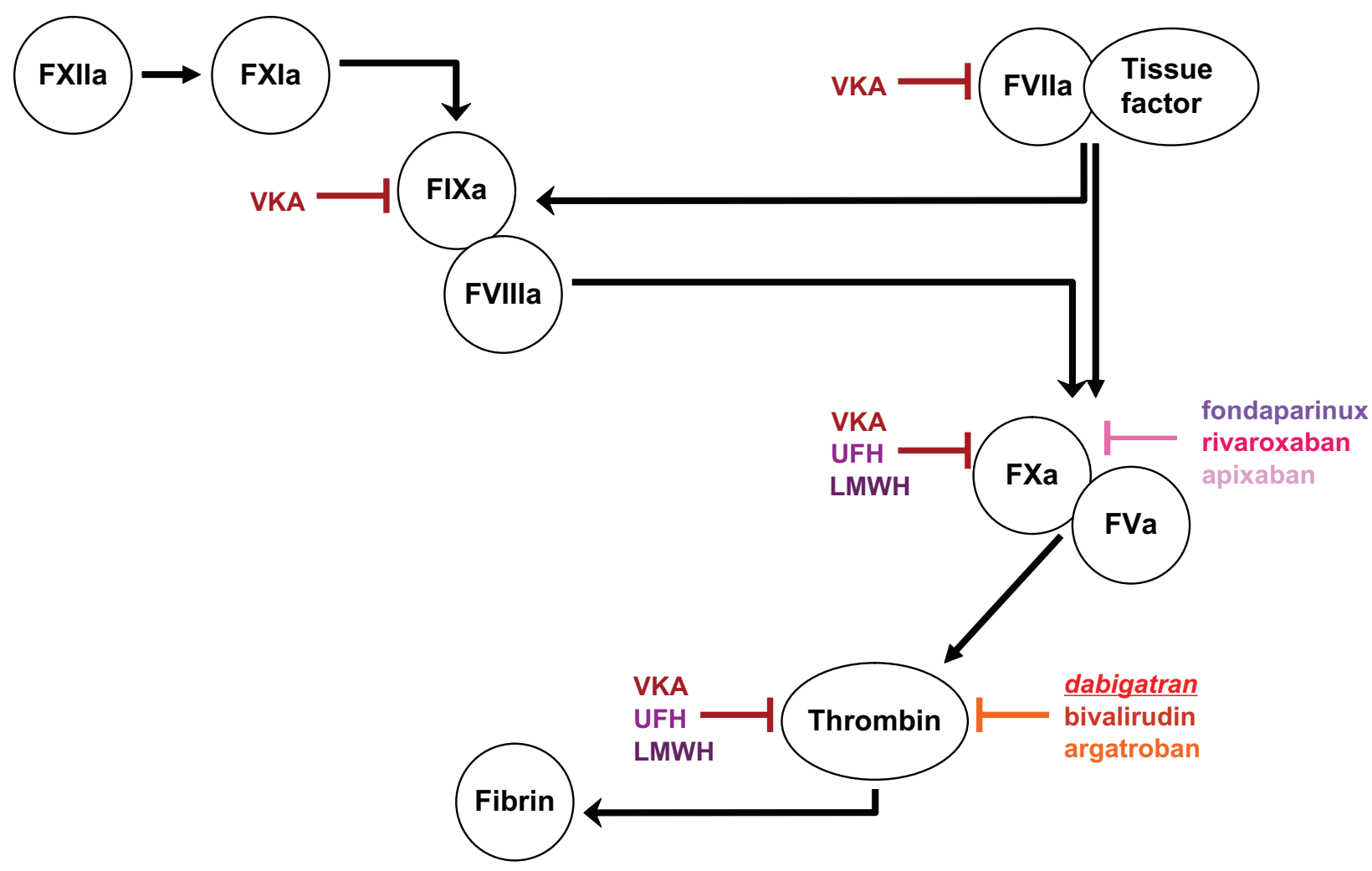

Figure I Targets of different anticoagulant agents (indicated in colored blocking arrows) in the coagulation cascade. Abbreviations: F, factor; LMWH, low-molecular-weight heparin; UFH, unfractionated heparin; VKA, vitamin K-antagonist.

reduce the risk of stroke in $\mathrm{AF}$ and the likelihood of recurrence in VTE. ${ }^{29}$ However, VKAs are limited by a narrow therapeutic window, with a resultant need for frequent monitoring and dosage adjustments. Other drawbacks of this class include drug and food interactions (eg, vitamin-K-rich foods), a well-documented incidence of major bleeding of $1 \%$ to $3 \%$, and delayed onset and offset of anticoagulant effect. ${ }^{28}$ Bleeding is the most common adverse event with VKAs. The incidence of major bleeding varies from less than $2 \%$ a year with care in an anticoagulation clinic to $4 \%$ to $5 \%$ a year with usual medical care. ${ }^{19,27}$

\section{Heparins}

Unfractionated heparin (UFH) has been used for the prevention and treatment of thrombosis since the 1930s. Lowmolecular-weight heparins (LMWHs), derived from UFH by depolymerization, were introduced in Europe in the 1980s. ${ }^{29}$ Because of several clinical advantages, LMWHs have gradually replaced UFH for most indications. However, UFH continues to be used during cardiovascular surgery and catheter-based interventional procedures. ${ }^{30}$

Heparins and heparinoids are parenteral agents, administered either intravenously or subcutaneously. The need for parenteral administration makes these agents both inconvenient and costly for long-term use, especially outside the hospital setting. ${ }^{15}$

Each LMWH product has a specific molecular weight distribution. This distribution determines its anticoagulant activity, duration of action, and renal clearance, so each agent is considered a unique drug. Indications for LMWHs vary - one product cannot always be substituted for another. LMWHs in current use globally include enoxaparin, dalteparin, nadroparin, tinzaparin, certoparin, reviparin, parnaparin and bemiparin. ${ }^{31}$

Bleeding is the most common adverse event with heparin therapy. Major bleeding occurs in $0.8 \%$ of patients receiving full-dose UFH, but is less frequent with lowdose subcutaneous heparin. LMWH has been reported to cause bleeding less frequently, but this finding has not been consistent across trials. Major bleeding occurs in less than $3 \%$ of patients and varies with product, indication, patient population, and dose. ${ }^{32}$

Although, hemorrhage is the most common and best recognized complication of heparin treatment, potentially more dangerous is the development of heparin-induced thrombocytopenia (HIT), especially HIT complicated by 
thrombosis syndrome (HITTS). All patients exposed to heparin, irrespective of the dose and route of administration, are at risk of developing HIT. This condition develops as the result of the formation of antibodies to the heparin-platelet factor 4 complex, which causes secondary activation of platelets, coagulation, and ultimately increased thrombin production. ${ }^{33}$

Danaparoid, available in several countries, is classified as a heparinoid. It is composed of sulfated glycosaminoglycans and can be used as an alternative to heparin in patients suffering from HIT. ${ }^{31}$

\section{Factor Xa inhibitors}

Fondaparinux, a pentasaccharide, is a synthetic indirect inhibitor of Factor Xa (FXa). Unlike heparin, fondaparinux does not inactivate thrombin or inhibit FXa bound in the prothrombinase complex and therefore does not completely inhibit FXa. Long-term use is limited by the requirement of subcutaneous injection. ${ }^{34}$

Bleeding is the most common adverse event with fondaparinux, with major bleeding occurring at about the same rate as seen with patients treated with LMWHs. Episodes of excessive bleeding due to fondaparinux may be treated effectively with Novoseven (recombinant coagulation factor VIIa). ${ }^{34}$

Orally available FXa-inhibitors, such as rivaroxaban and apixaban, are currently being investigated in large Phase II and III trials. Rivaroxaban (Xarelto ${ }^{\circledR}$; Bayer Schering Pharma) is already approved for the primary prevention of VTE after orthopedic surgery in adults in Canada as well as the European Union.

\section{Direct thrombin inhibitors}

Parenteral direct thrombin inhibitors are used during percutaneous coronary interventions (PCIs), and to treat or prevent thrombosis in patients with HIT. Three such medications are in current clinical use - lepirudin, bivalirudin, and argatroban. These drugs differ with respect to thrombin binding sites, reversibility, pharmacology, and specific indications. ${ }^{35}$

The first oral direct thrombin inhibitor available for clinical use was ximelagatran. This medication represented a major advance over existing oral anticoagulants (eg, VKAs) because it did not require anticoagulant monitoring or dose adjustments. In clinical VTE prevention and treatment, ximelagatran was either more effective than or comparable to warfarin. However, safety monitoring revealed liver toxicity in $6 \%$ of patients. For this reason, use of the drug was discontinued in $2006 .{ }^{36}$
Advantages and disadvantages of the oral direct thrombin inhibitor dabigatran etexilate will be described in the following sections.

\section{Dabigatran etexilate- pharmacology, pharmacokinetics, and safety}

Dabigatran etexilate (Pradaxa ${ }^{\circledR}$ in Europe, Pradax ${ }^{\circledR}$ in Canada; Boehringer Ingelheim) is a small molecule prodrug which does not exhibit any pharmacological activity. After oral administration, dabigatran etexilate is rapidly absorbed and converted to dabigatran by esterase-catalyzed hydrolysis in plasma and in the liver. The prodrug contains micropellets with dabigatran etexilate around a tartaric acid core, since a low $\mathrm{pH}$ is necessary for optimal drug absorption. ${ }^{37}$

Dabigatran is a potent, competitive, reversible direct thrombin inhibitor. Since thrombin (serine protease) enables the conversion of fibrinogen into fibrin during the coagulation cascade, its inhibition prevents the development of thrombus. Dabigatran also inhibits free thrombin, fibrin-bound thrombin and thrombin-induced platelet aggregation. ${ }^{38}$

Dabigatran prolongs the activated partial thromboplastin time (aPTT). In patients who are bleeding, aPTT tests may be useful in identifying excessive anticoagulant activity, despite the aPTT being less sensitive to the activity of dabigatran at supratherapeutic levels. If available, thrombin time and ecarin clotting time may be more sensitive tests to evaluate the anticoagulant effects of dabigatran. Prothrombin time (INR, international normalized ratio) is prolonged by dabigatran but is less sensitive than thrombin time and ecarin clotting time. ${ }^{39}$

After oral administration of dabigatran etexilate in healthy volunteers, the pharmacokinetic profile of dabigatran in plasma is characterized by a rapid increase in plasma concentration with maximum concentration $\left(\mathrm{C}_{\max }\right)$ attained between 0.5 and 2.0 hours post-administration. After $\mathrm{C}_{\max }$, plasma concentrations of dabigatran show a biexponential decline with a mean terminal half-life of 14 to 17 hours in young healthy volunteers, and 12-14 hours in elderly subjects. The half-life is independent of dose. Food does not affect the bioavailability of dabigatran but delays the time to peak plasma concentrations by 2 hours. The absolute bioavailability of dabigatran following oral administration of dabigatran etexilate is approximately $6.5 \% .^{40}$

Dabigatran is eliminated largely unchanged in the urine, at a rate of approximately $100 \mathrm{~mL} / \mathrm{min}$ corresponding to the glomerular filtration rate. Low (34\% to $35 \%$ ) concentration independent binding of dabigatran to human plasma proteins was observed. The exposure (area under the curve = AUC) of dabigatran after the oral administration of dabigatran 
etexilate is approximately 2.7 -fold higher in volunteers with moderate renal insufficiency (creatinine clearance $=\mathrm{CrCL}$ between 30 to $50 \mathrm{~mL} / \mathrm{min}$ ) than in those without renal insufficiency. In a small number of volunteers with severe renal insufficiency (CrCL 10 to $30 \mathrm{~mL} / \mathrm{min}$ ), the exposure (AUC) to dabigatran was approximately 6 times higher and the half-life approximately 2 times longer than that observed in a population without renal insufficiency. ${ }^{41}$ Specific pharmacokinetic studies with elderly subjects showed increases of $40 \%$ to $60 \%$ in the exposure (AUC) and of more than $25 \%$ in $\mathrm{C}_{\max }$ compared to young subjects. Population-based pharmacokinetic studies have evaluated the pharmacokinetics of dabigatran after repeated doses in patients (up to 88 years). The observed increase in dabigatran exposure (AUC) correlated with age-related reductions in CrCL. ${ }^{41}$

No change in dabigatran exposure (AUC) was seen in 12 subjects with moderate hepatic insufficiency (ChildPugh B) compared to 12 controls. Body weight had a minor effect on plasma clearance of dabigatran resulting in higher exposure (AUC) in patients with low body weight. ${ }^{42}$ There were no differences in the phase 3 clinical studies for efficacy and safety data between men and women. Drug exposure (AUC) in female patients is about $40 \%$ to $50 \%$ higher than in male patients, but no dose adjustment is recommended. Ethnic origin does not appear to affect the pharmacokinetics of dabigatran in a clinically relevant manner. However, no pharmacokinetic data in black patients are available. ${ }^{43}$

In vitro interaction studies did not show any inhibition or induction of the principal isoenzymes of cytochrome P450. ${ }^{44}$ This has been confirmed by in vivo studies with healthy volunteers, who did not show any interaction between this treatment and atorvastatin (CYP3A4), digoxin (P-gp transporter interaction) or diclofenac (CYP2C9). However, dabigatran is not without important drug interactions. P-glycoprotein inhibitors (including verapamil, amiodarone, and especially quinidine) raise dabigatran serum concentrations considerably. For example, dabigatran exposure in healthy subjects was increased by $60 \%$ in the presence of amiodarone. ${ }^{42}$

The population pharmacokinetic analysis of co-medication effects supports the use of antacids and gastric acids suppressants without dose adjustment of dabigatran etexilate in patients, and revealed the absence of dabigatran drug interactions with the most commonly used drugs in the study population. ${ }^{43}$

\section{Dabigatran etexilate in the prevention of deep vein thrombosis}

For more than 20 years, routine preventive anticoagulant therapy has been the standard of care for major orthopedic surgery. Despite the well-recognized risks of VTE in hospitalized patients, there remains a low rate of appropriate prophylaxis. $^{5}$

Major orthopedic surgery involving the lower extremity hip or knee replacement surgery or hip fracture surgery - is associated with a high risk of VTE. Without prophylaxis, rates of DVT range from $40 \%$ to $60 \%$ when assessed by venography 7 to 14 days after surgery. Routine ventilationperfusion scans in patients following hip or knee arthroplasty revealed pulmonary emboli in $3 \%$ to $28 \%$ of patients. ${ }^{3}$ Most cases of symptomatic VTE manifest after discharge from the hospital, and are the most common reason for readmission following orthopedic procedures. ${ }^{1,5}$

Patients who have had total hip replacement and no VTE by venography at discharge continue to be at risk of late-onset VTE up to 35 days after surgery. ${ }^{2}$ Current recommendations regarding the duration of anticoagulation treatment following hip and knee surgery are based on this understanding of the chronology of VTE. ${ }^{7}$

In 2 large randomized, parallel group, double-blind, doseconfirmatory trials, ${ }^{45,46}$ patients undergoing elective major orthopedic surgery (one for knee replacement surgery and one for hip replacement surgery) received dabigatran etexilate $75 \mathrm{mg}$ or $110 \mathrm{mg}$ within 1 to 4 hours of surgery followed by 150 or $220 \mathrm{mg}$ daily thereafter, hemostasis having been secured, or enoxaparin $40 \mathrm{mg}$ on the day prior to surgery and daily thereafter. In the RE-MODEL trial (knee replacement) treatment was for 6 to 10 days and in the RE-NOVATE trial (hip replacement) for 28 to 35 days. Totals of 2076 patients (knee) and 3494 (hip) were treated respectively. The results of RE-MODEL with respect to the primary end-point, total VTE including asymptomatic DVT plus all-cause mortality, showed that the antithrombotic effect of both doses of dabigatran etexilate were statistically non-inferior to that of enoxaparin. Similarly, total VTE including asymptomatic DVT and all-cause mortality constituted the primary endpoint for RE-NOVATE. Again dabigatran etexilate at both daily doses was statistically non-inferior to enoxaparin $40 \mathrm{mg}$ daily.

However, non-inferiority was not established in a third randomized, parallel group, double-blind trial(RE-MOBILIZE), in which patients undergoing elective total knee surgery received dabigatran etexilate $75 \mathrm{mg}$ or $110 \mathrm{mg}$ within 6 to 12 hours of surgery followed by $150 \mathrm{mg}$ and $220 \mathrm{mg}$ daily thereafter. The treatment duration was 12 to 15 days. In total 2615 patients were randomized and 2596 were treated. The comparator dosage of enoxaparin was $30 \mathrm{mg}$ twice daily according to common recommendations in the US. 
There were no statistical differences in bleeding between the comparators. ${ }^{47}$ Mostly due to these findings dabigatran etexilate did not get approved for treatment in North America at this point by the US Food and Drug Administration.

A randomized, parallel group, double-blind, placebocontrolled phase II study in Japanese patients ${ }^{48}$ in which dabigatran etexilate was administered at $110 \mathrm{mg}, 150 \mathrm{mg}$, and $220 \mathrm{mg}$ the day after elective total knee replacement surgery, showed a clear dose response relationship for the efficacy of dabigatran etexilate and a placebo like bleeding profile. In RE-MODEL and RENOVATE the randomization to the respective study medication was done pre-surgery, and in the RE-MOBILIZE and the Japanese trial the randomization was performed post-surgery. This is of note especially in the safety evaluation of these trials.

In the recently published RE-COVER trial, dabigatran etexilate (150 mg twice daily) was as effective and safe as warfarin (adjusted INR of 2.0 to 3.0) in patients suffering from acute VTE. ${ }^{49}$ Further studies are currently recruiting patients to investigate the efficacy and safety of dabigatran in the prevention of secondary VTE (RE-MEDY and RE-SONATE).

\section{Dabigatran etexilate in stroke prevention and management of atrial fibrillation}

Cardioembolic stroke is one of the main complications of $\mathrm{AF}$, and occurs when stagnant blood in the fibrillating atrium forms a thrombus that then embolizes to the cerebral circulation, blocking arterial blood flow and causing ischemic injury. The incidence of stroke in patients with nonvalvular AF is between 2- and 7-fold greater than in the general population. For patients with AF caused by valvular disease, the risk of stroke increases 17 -fold..$^{50}$

A systematic review of 6 cohorts of AF patients identified three other independent risk factors in addition to age: prior history of stroke or transient ischemic attack, history of hypertension, and diabetes. ${ }^{51}$ Several scoring systems are available to help clinicians estimate the stroke risk in AF. One popular, well-validated risk assessment tool is the $\mathrm{CHADS}_{2}$-Score (Table 2). ${ }^{52}$

The recently presented RE-LY trial, ${ }^{53}$ which compared two fixed doses of dabigatran etexilate (110 mg and $150 \mathrm{mg}$ ) administered twice daily to warfarin, has major implications for prevention of stroke, thromboembolic complications, and life-threatening hemorrhagic complications. The trial randomly assigned 18113 patients who had AF and a concomitant risk of stroke. Rates of the primary outcome (stroke or systemic embolism) were $1.69 \%$ per year in the warfarin group compared with $1.53 \%$ per year in the group that received $110 \mathrm{mg}$ of dabigatran (relative risk with dabigatran, $0.91 ; 95 \%$ confidence interval $[\mathrm{CI}] 0.74$ to $1.11 ; P<0.001$ for non-inferiority), and $1.11 \%$ per year in the group that received $150 \mathrm{mg}$ of dabigatran (relative risk, 0.66; 95\% CI 0.53 to $0.82 ; P<0.001$ for superiority).

The rate of major bleeding was $3.36 \%$ per year in the warfarin group, as compared with $2.71 \%$ per year in the group receiving $110 \mathrm{mg}$ of dabigatran $(P=0.003)$ and $3.11 \%$ per year in the group receiving $150 \mathrm{mg}$ of dabigatran $(P=0.31)$. Further, the rate of hemorrhagic stroke was $0.38 \%$ per year in the warfarin group, compared with $0.12 \%$ per year with $110 \mathrm{mg}$ of dabigatran $(P<0.001)$ and $0.10 \%$ per year with $150 \mathrm{mg}$ of dabigatran $(P<0.001)$.

No real advantage was seen in mortality rate, which was $4.13 \%$ per year in the warfarin group, $3.75 \%$ per year with $110 \mathrm{mg}$ of dabigatran $(P=0.13)$ and $3.64 \%$ per year with $150 \mathrm{mg}$ of dabigatran $(P=0.051)$.

In sum, dabigatran administered at a dose of $110 \mathrm{mg}$ twice daily was associated with rates of stroke and systemic embolism that were similar to those seen with warfarin, but with lower rates of hemorrhage, while dabigatran at $150 \mathrm{mg}$ twice daily was associated with lower rates of stroke and systemic embolism but similar rates of major hemorrhage.

Likely due to the controlled trial setting, the rate of bleeding in the warfarin group was slightly lower $(3.36 \%$ per year) than is usually seen in real-world ambulatory care of

Table $2 \mathrm{CHADS}_{2}$ score

\begin{tabular}{lll}
\hline C: & Congestive heart failure & $=1$ point \\
H: & Hypertension (systolic $>160 \mathrm{mmHg})$ & $=1$ point \\
A: & Age $>75$ years & $=1$ point \\
D: & Diabetes & $=1$ point \\
S: & Prior transient ischemic attack or stroke & $=2$ points
\end{tabular}

CHADS $_{2}$ score $\quad$ Adjusted stroke rate ${ }^{a}(\%)$ ( $95 \%$ confidence interval)

\begin{tabular}{lc}
\hline 0 & I.9 $(I .2-3.0)$ \\
$I$ & $2.8(2.0-3.8)$ \\
2 & $4.0(3 . I-5 . I)$ \\
3 & $5.9(4.6-7.3)$ \\
4 & $8.5(6.3-I I . I)$ \\
5 & $I 2.5(8.2-I 7.5)$ \\
6 & I $8.2(I 0.5-27.4)$ \\
\hline Notes: The annual stroke rates for patients suffering from AF according to the \\
CHADS Score. Patients with a CHADS Score of 0 are considered to have a low \\
risk for cardioembolic stroke. Patients with a score of I-2 are classified as having \\
a moderate risk for stroke and should be medically treated with an antithrombotic \\
agent. Patients with a score of 3 or higher have a high risk for thromboembolic \\
complications and require optimal antithrombotic treatment as well. \\
aThe adjusted stroke rate is the expected stroke rate per I00 person-years derived \\
from the multivariable model assuming that aspirin is not taken.
\end{tabular}


anticoagulated patients. It is possible that, if used in patients outside of a clinical trial, bleeding complications will occur even more infrequently with dabigatran than with warfarin.

Surprisingly, the annualized rates for myocardial infarction (MI) were $0.72 \%$ for low-dose dabigatran, $0.74 \%$ for high-dose dabigatran, and $0.53 \%$ for warfarin. The risk trended higher for low-dose dabigatran at 1.35 (95\% CI 0.98 to $1.87 ; P=0.07$ ) and was significantly higher for in the highdose group at 1.38 (95\% CI 1.00 to $1.91 ; P=0.048)$. This somewhat unexpected finding leads to the hypothesis that warfarin is more effective in reducing MI than dabigatran at this particular aspect of antithrombotic therapy. However, the absolute rates of MI were quite low, and the effect of dabigatran in reducing stroke and intracerebral bleedings far outweighed the small increase in MI rate.

Rates of dyspepsia (including abdominal pain) were increased with dabigatran (11.8\% in the 110-mg group and $11.3 \%$ in the 150 -mg group) as compared with warfarin $(5.8 \%)$, and it may have contributed to the greater second-year rate of dropout in patients taking dabigatran (approximately $21 \%)$ as compared with warfarin $(16.6 \%) .{ }^{54}$

\section{Prospective indications for dabigatran etexilate Mechanical heart valve thrombosis}

Lifelong OAC therapy is required for the prevention of thromboembolic events after implantation of an artificial heart valve. Thromboembolism and anticoagulant-related bleedings account for $\approx 75 \%$ of all complications experienced by heart valve recipients $(2 \%$ to $9 \%$ of patients per year). In preparation for surgical procedures, OAC treatment must be interrupted. The gold standard for adjusting oral to parenteral anticoagulation treatment in patients at a high risk for developing thromboembolic complications is intravenous administration of $\mathrm{UFH},{ }^{25,26}$ which needs to be continuously infused to ensure an adequate effect. However, anticoagulation with UFH is associated with an increased rate of complications. These complications are related to low bioavailability, short half-life, platelet activation, and interindividual differences regarding optimal dosage of UFH. ${ }^{27}$ Although most guidelines favor intravenous administration of UFH, LMWH (eg, enoxaparin, dalteparin, and tinzaparin) represent an additional option for bridging therapy. ${ }^{25,26}$

A new anticoagulant that more closely meets the criteria for optimal antithrombotic efficacy could improve the quality of care. Such an advance also might help overcome gaps between evidence-based treatment recommendations and clinical practice.
Studies in patients with a strong indication for anticoagulant therapy because of high thromboembolic risk without adequate protection, such as patients with a mechanical heart valve, are not scheduled in the early phase of development of new antithrombotics. Inappropriate dosing would lead to unacceptable outcomes in this high-risk population. A new agent such as dabigatran etexilate could be a welcome addition for improving care in these patients. Future prospective and randomized studies in humans should address the question of whether orally administered dabigatran etexilate is as effective as UFH, LMWH, and VKAs in preventing thrombus formation on mechanical heart valves. Notably, large, randomized, prospective trials are also lacking for LMWH as regards prevention of mechanical heart valve thrombosis.

Less thrombogenic materials and improved valve and hinge designs may reduce the propensity for thrombus formation and the need for aggressive anticoagulation. This might reverse the present trend to increased use of bioprosthetic valves. ${ }^{55}$ The step from preclinical in vitro and in vivo testing to widespread clinical use is hampered by the lack of good predictive animal models and always entails a certain risk.

\section{Acute coronary syndrome and percutaneous coronary intervention}

Risk assessment is a key step in the emergency management of patients with ACS. Stratifying patients by likelihood of morbidity and mortality guides management decisions. A range of risk-scoring systems have been devised to enable clinicians to select the appropriate antithrombotic, antiplatelet and/or fibrinolytic therapy. ${ }^{27}$

Patients with complete occlusion of a coronary artery can often be identified by ST-segment elevation on electrocardiogram. This group, representing approximately one in three patients presenting with ACS, should receive prompt reperfusion treatment with fibrinolytic therapy or PCI. The remaining two-thirds of ACS patients do not have ST-segment elevation on initial ECG, and require further risk stratification. ${ }^{22}$

Because atherosclerotic plaque is often present throughout the arterial tree, patients who survive an episode of ACS live with an ongoing risk of a recurrent acute cardiovascular event, such as MI, sudden cardiac death, or stroke. ${ }^{56}$ For patients who receive a stent during revascularization, there is an additional risk of in-stent thrombosis. ${ }^{22}$

A wide range of treatments are available to protect the heart and to decrease the propensity for recurrent atherothrombosis in survivors of ACS. Antiplatelet agents such as 
ASA and clopidogrel are used for up to 12 months after ACS to prevent a recurrent event. ${ }^{22}$ For immediate antithrombotic therapy a wide range of agents are available. So far all of them need to be administered intravenously. DTIs currently available for intravenous administration that are successfully and primarily used in the treatment of HIT as well as PCI are lepirudin, bivalirudin, and argatroban. ${ }^{57}$ DTIs are potentially ideal agents for PCI and ACS patients due to their antithrombotic and antiplatelet properties.

Dabigatran is currently being studied in a randomized, open-label phase-II trial in patients undergoing elective PCI (ClinicalTrials.gov Identifier: NCT00818753). The purpose of the study is to assess whether two doses of dabigatran etexilate (110 mg twice daily and $150 \mathrm{mg}$ twice daily) compared with UFH, both in addition to a standard dual antiplatelet regimen, provide sufficient anticoagulation in the setting of elective PCI.

Currently usage of VKAs in patients after ACS has very limited indications as an add-on therapy to antiplatelet drugs such as clopidogrel and aspirin. These indications include patients with AF and at high risk for stroke, mechanical heart valve recipients, and patients with a left ventricular aneurysm and/or thrombus formation in the left ventricular cavity. Triple therapy with antiplatelet drugs and VKAs is associated with a high risk of bleeding. The frequency of such events in reported series varies, with up to $21 \%$ of patients needing a transfusion. Bleeding events typically involve the gastrointestinal tract. ${ }^{58}$ Evidence suggests that this frequency increases with longer duration of triple therapy, which directly correlates with bleeding risk and might influence mortality in follow-up after PCI. In particular, the relative risk of major bleeding in patients receiving triple therapy is 3- to 5-fold higher than that observed in patients receiving dual antiplatelet therapy alone. The increase in bleeding events is confounded by the fact that patients receiving triple therapy are typically older and have multiple co-morbidities. Limited use of triple therapy (for 1 month) is associated with at least a 2-fold lower risk of major bleeding compared with prolonged use ( $\geq 6$ months). ${ }^{58}$ Dabigatran etexilate may have a role as an acceptable substitute for VKAs in these high risk patients, but this has not yet been studied.

\section{Conclusions}

Recent advances in antithrombotic therapy have the potential to significantly affect the management of patients with thromboembolic disease and disease risk. The development of new orally available agents has already changed the field of anticoagulation beyond the once gold standard of VKAs, and will undoubtedly result in a paradigm shift in the management of these diseases. Warfarin has proven to be very effective for the primary prevention of stroke and AF, as well as for the long-term management of patients with a whole spectrum of thromboembolic-related illnesses. However, its significant limitations have been discussed throughout the review.

These limitations have led to a search for new anticoagulant drugs, a quest made easier by improved understanding of coagulation and targeted inhibition of specific coagulation factors. Not surprisingly, since FXa is the key coagulation factor lying at the crossroads of intrinsic and extrinsic coagulation, a significant interest exists in developing drugs which specifically target activated FXa. Moreover, the inhibition of thrombin may be the most efficient way of inhibiting coagulation, giving rise to the development of drugs which specifically target it. ${ }^{59}$ Although it is currently not clear which mechanism will give rise to better clinical outcomes, both FXa and thrombin are likely to be excellent targets for inhibition with new anticoagulant drugs.

FXa inhibition appears to be the more promising avenue, as Factor $\mathrm{X}$ has fewer functions outside coagulation compared with thrombin. Also, Factor X is associated with a shallower dose response curve, suggesting that Xa inhibitors may have a wider therapeutic window compared to thrombin inhibitors. In contrast, thrombin inhibitors may allow other actions of thrombin to occur, such as activation of the anti-coagulant protein C. ${ }^{60}$

New oral anticoagulants that are currently in advanced stages of clinical trials offer the potential to revolutionize long-term anticoagulant therapy. Besides dabigatran etexilate these agents are rivaroxaban, apixaban, and edoxaban, all of which inhibit FXa.

Dabigatran etexilate has proven to be a competitive antithrombotic. Potential drawbacks of the drug include the higher occurrence of MIs in the RELY study (in comparison to warfarin), the inferiority compared to the North American enoxaparin regime in the RE-MOBILIZE study, as well as the lack of a readily available antidote or an established anticoagulant monitoring for the detection of a potential non-compliance in standard clinical practice. So far recommendations for dabigatran use in special populations, like renal impairment or failure and application during pregnancy, are also missing. One has to be cautious to only use dabigatran etexilate in the currently approved indication in the European Union and Canada, the primary prevention of VTEs in adults after elective total hip or knee replacement surgery. Further approvals for other countries as well as other indications are pending. 
A drug that will prevent coagulation without promoting bleeding has yet to be found. However, molecules that contribute to thrombosis continue to be identified, and these could be new targets for the next generation of antithrombotic therapy. More immediately, recent studies of new antiplatelet drugs (such as prasugrel) and new anticoagulant drugs (such as dabigatran etexilate) suggest that more options will soon be available for the treatment of thrombosis. Furthermore, the ability to identify patients at increased risk of thrombosis by measuring the concentrations of circulating factors, such as tissue factor, might allow more effective use of prophylaxis. Finally, "personalized medicine" is on the horizon, which could allow customized dosing of antithrombotic drugs rather than the current "one dose fits all" strategy.

\section{Disclosures}

The authors have no conflicts of interest to declare.

\section{References}

1. Geerts WH, Bergqvist D, Pineo GF, et al. Prevention of Venous Thromboembolism: American College of Chest Physicians (ACCP) Evidence-Based Clinical Practice Guidelines (8th Edition). Chest. 2008;381S-453S.

2. Moll S, Mackman N. Venous thromboembolism: a need for more public awareness and research into mechanisms. Arterioscler Thromb Vasc Biol. 2008;28:367-369.

3. Cohen AT, Tapson VF, Bergmann JF, et al; ENDORSE Investigators. Venous thromboembolism risk and prophylaxis in the acute hospital care setting (ENDORSE study): a multinational cross-sectional study. Lancet. 2008;371:387-394.

4. Lapostolle F, Surget V, Borron SW, et al. Severe pulmonary embolism associated with air travel. $N$ Engl J Med. 2001;345:779-783.

5. Francis CW. Clinical practice. Prophylaxis for thromboembolism in hospitalized medical patients. N Engl J Med. 2007;356:1438-1444.

6. Heit JA, Silverstein MD, Mohr DN, Petterson TM, O'Fallon WM, Melton LJ 3rd. Risk factors for deep vein thrombosis and pulmonary embolism: a population-based case-control study. Arch Intern Med. 2000;160:809-815.

7. White RH. The epidemiology of venous thromboembolism. Circulation. 2003;107:I4-I8.

8. Kearon C. Natural history of venous thromboembolism. Circulation. 2003;107:I22-I30.

9. Rodger MA, Kahn SR, Wells PS, et al. Identifying unprovoked thromboembolism patients at low risk for recurrence who can discontinue anticoagulant therapy. CMAJ. 2008;179:417-426.

10. Kahn SR. The post-thrombotic syndrome: the forgotten morbidity of deep venous thrombosis. J Thromb Thrombolysis. 2006;21:41-48.

11. Haenen JH, Janssen MC, van Langen H, et al. The postthrombotic syndrome in relation to venous hemodynamics, as measured by means of duplex scanning and strain-gauge plethysmography. J Vasc Surg. 1999;29:1071-1076.

12. Prandoni P, Lensing AW, Cogo A, et al. The long-term clinical course of acute deep venous thrombosis. Ann Intern Med. 1996;125:1-7.

13. Pengo V, Lensing AW, Prins MH, et al. Incidence of chronic thromboembolic pulmonary hypertension after pulmonary embolism. $N$ Engl J Med. 2004;350:2257-2264.

14. Fedullo PF, Auger WR, Kerr KM, Rubin LJ. Chronic thromboembolic pulmonary hypertension. $N$ Engl J Med. 2001;345:1465-1472.
15. Singer DE, Albers GW, Dalen JE, et al; American College of Chest Physicians. Antithrombotic therapy in atrial fibrillation: American College of Chest Physicians Evidence-Based Clinical Practice Guidelines (8th Edition). Chest. 2008;133:546-592.

16. Lip G, Nieuwlaat R, Pisters R, Lane D, Crijns H. Refining clinical risk stratification for predicting stroke and thromboembolism in atrial fibrillation using a novel risk factor based approach: The Euro Heart Survey on Atrial Fibrillation. Chest. 2010;137:263-272.

17. Schnabel RB, Sullivan LM, Levy D, et al. Development of a risk score for atrial fibrillation (Framingham Heart Study): a community-based cohort study. Lancet. 2009;373:739-745.

18. Hylek EM. Antithrombotic prophylaxis in elderly patients with atrial fibrillation. Semin Thromb Hemost. 2009;35:548-553.

19. Levi M, Hobbs FD, Jacobson AK, et al. Improving antithrombotic management in patients with atrial fibrillation: current status and perspectives. Semin Thromb Hemost. 2009;35:527-542.

20. Pisters R, de Vos CB, Nieuwlaat R, Crijns HJ. Use and underuse of oral anticoagulation for stroke prevention in atrial fibrillation: old and new paradigms. Semin Thromb Hemost. 2009;35:554-559.

21. Levi M, de Peuter OR, Kamphuisen PW. Management strategies for optimal control of anticoagulation in patients with atrial fibrillation. Semin Thromb Hemost. 2009;35:560-567.

22. Anderson JL, Adams CD, Antman EM, et al; American College of Cardiology; American Heart Association Task Force on Practice Guidelines. ACC/AHA 2007 guidelines for the management of patients with unstable angina/non-ST-Elevation myocardial infarction: a report of the American College of Cardiology/American Heart Association Task Force on Practice Guidelines. J Am Coll Cardiol. 2007;50:1-157.

23. Vickrey BG, Rector TS, Wickstrom SL, et al. Occurrence of secondary ischemic events among persons with atherosclerotic vascular disease. Stroke. 2002;33:901-906.

24. Cannegieter SC, Torn M, Rosendaal FR. Oral anticoagulant treatment in patients with mechanical heart valves: how to reduce the risk of thromboembolic and bleeding complications. J Intern Med. 1999;245:369-374.

25. American College of Cardiology/American Heart Association Task Force on Practice Guidelines; Society of Cardiovascular Anesthesiologists; Society for Cardiovascular Angiography and Interventions; Society of Thoracic Surgeons, Bonow RO, Carabello BA, Kanu C, et al. ACC/AHA guidelines for the management of patients with valvular heart disease. A report of the American College of Cardiology/American Heart Association Task Force on Practice Guidelines (writing committee to revise the 1998 Guidelines for the Management of Patients With Valvular Heart Disease. Circulation. 2006;114:84-231.

26. Vahanian A, Baumgartner H, Bax J, et al; Task Force on the Management of Valvular Hearth Disease of the European Society of Cardiology; ESC Committee for Practice Guidelines. Guidelines on the management of valvular heart disease. Eur Heart J. 2007;28: 230-268.

27. Hirsh J, Guyatt G, Albers GW, Harrington R, Schünemann HJ; American College of Chest Physician. Antithrombotic and thrombolytic therapy: American College of Chest Physicians Evidence-Based Clinical Practice Guidelines (8th Edition). Chest. 2008;133:110-112.

28. Eikelboom JW, Weitz JI. A replacement for warfarin: the search continues. Circulation. 2007;116:131-133.

29. Hirsh J, O'Donnell M, Eikelboom JW. Beyond unfractionated heparin and warfarin: current and future advances. Circulation. 2007;116: $552-560$.

30. Frydman A. Low-molecular-weight heparins: an overview of their pharmacodynamics, pharmacokinetics and metabolism in humans. Haemostasis. 1996;26:24-38.

31. Bick RL, Frenkel EP, Walenga J, Fareed J, Hoppensteadt DA. Unfractionated heparin, low molecular weight heparins, and pentasaccharide: basic mechanism of actions, pharmacology, and clinical use. Hematol Oncol Clin North Am. 2005;19:1-51.

32. Vesely MR, Kelemen MD. Cardiac risk assessment: matching intensity of therapy to risk. Cardiol Clin. 2006;24:67-78. 
33. Warkentin TE, Greinacher A, Koster A, and Lincoff AM. Treatment and Prevention of Heparin-Induced Thrombocytopenia: American College of Chest Physicians (ACCP) Evidence-Based Clinical Practice Guidelines (8th Edition). Chest. 2008;340S-380S.

34. Chaturvedi V, Karthikeyan G. Fondaparinux in acute coronary syndromes. Expert Opin Drug Metab Toxicol. 2009;5:1615-1623.

35. Harenberg J, Wehling M. Current and future prospects for anticoagulant therapy: inhibitors of factor Xa and factor IIa. Semin Thromb Hemost. 2008;34:39-57.

36. Haas S. New anticoagulants - towards the development of an "ideal" anticoagulant. Vasa. 2009;38:13-29.

37. Wienen W, Stassen JM, Priepke H, Ries UJ, Hauel N. Antithrombotic and anticoagulant effects of the direct thrombin inhibitor dabigatran, and its oral prodrug, dabigatran etexilate, in a rabbit model of venous thrombosis. J Thromb Haemost. 2007;5:1237-1242.

38. Stangier J, Rathgen K, Stähle H, Gansser D, Roth W. The pharmacokinetics, pharmacodynamics and tolerability of dabigatran etexilate, a new oral direct thrombin inhibitor, in healthy male subjects. Br J Clin Pharmacol. 2007;64:292-303.

39. Eriksson BI, Smith H, Yasothan U, Kirkpatrick P. Dabigatran etexilate Nat Rev Drug Discov. 2008;7:557-558.

40. Haas S. New oral Xa and IIa inhibitors: updates on clinical trial results J Thromb Thrombolysis. 2008;25:52-60.

41. Wolowacz SE, Roskell NS, Plumb JM, et al. Economic evaluation of dabigatran etexilate for the preventionof venous thromboembolism in patients aged over 75 years or with moderate renal impairment undergoing total knee or hip replacement. Thromb Haemost. 2010;103:360-371.

42. Stangier J, Clemens A. Pharmacology, pharmacokinetics, and pharmacodynamics of dabigatran etexilate, an oral direct thrombin inhibitor Clin Appl Thromb Hemost. 2009 Aug 19. [Epub ahead of print].

43. Dahl OE. Dabigatran etexilate: A new oral thrombin inhibitor. therapy 2008;5:1-11.

44. Wienen W, Stassen JM, Priepke H, Ries UJ, Hauel N. In-vitro profile and ex-vivo anticoagulant activity of the direct thrombin inhibitor dabigatran and its orally active prodrug, dabigatran etexilate. Thromb Haemost. 2007;98:155-162.

45. Eriksson BI, Dahl OE, Rosencher N, et al; RE-MODEL Study Group. Oral dabigatran etexilate vs subcutaneous enoxaparin for the prevention of venous thromboembolism after total knee replacement: the REMODEL randomized trial. J thromb Haemost. 2007;5:2178-2185.

46. Eriksson BI, Dahl OE, Rosencher N, et al; RE-NOVATE Study Group. Dabigatran etexilate versus enoxaparin for prevention of venous thromboembolism after total hip replacement: a randomised, double-blind, non-inferiority trial. Lancet. 2007;370:949-956.
47. Ginsberg JS, Davidson BL, Comp PC, et al. RE-MOBILIZE Writing Committee. Oral thrombin inhibitor dabigatran etexilate vs North American enoxaparin regimen for prevention of venous thromboembolism after knee arthroplasty surgery. J Arthroplasty. 2009;24:1-9.

48. Fuji T, Fuijita S, Ujihira T, Sato T. Dabigatran etexilate prevents venous thromboembolism after total knee arthroplasty in Japanese patients, with a safety profile comparable to placebo. J Arthroplasty. 2009 Oct 24 . [Epub ahead of print].

49. Schulman S, Kearon C, Kakkar AK, et al. Dabigatran versus warfarin in the treatment of acute venous thromboembolism. $N$ Engl J Med. 2009;361:2342-2352.

50. Qureshi AI, Feldmann E, Gomez CR, et al. Intracranial atherosclerotic disease: an update. Ann Neurol. 2009;66:730-738.

51. Hart RG, Pearce LA, Aguilar MI. Meta-analysis: antithrombotic therapy to prevent stroke in patients who have nonvalvular atrial fibrillation. Ann Intern Med. 2007;146:857-867.

52. Gage BF, Waterman AD, Shannon W, Boechler M, Rich MW, Radford MJ. Validation of clinical classification schemes for predicting stroke: results from the National Registry of Atrial Fibrillation. JAMA. 2001;285:2864-2870.

53. Connolly SJ, Ezekowitz MD, Yusuf S, et al. RE-LY Steering Committee and Investigators. Dabigatran versus Warfarin in Patients with Atrial Fibrillation. N Engl J Med. 2009;361:1139-1151.

54. Gage BF. Can we rely on RE-LY? N Engl J Med. 2009;361: 1200-1202.

55. Herijgers $P$, Verhamme P. Improving the quality of anticoagulant therapy in patients with mechanical heart valves: what are we waiting for? Eur Heart J. 2007;28:2424-2426.

56. Viles-Gonzalez JF, Fuster V, Badimon JJ. Atherothrombosis: a widespread disease with unpredictable and life-threatening consequences. Eur Heart J. 2004;25:1197-1207.

57. Turpie AG. Direct Factor Xa and direct thrombin inhibitors: a clinical trial update. Curr Opin Drug Discov Devel. 2009;12:497-508.

58. Holmes DR Jr, Kereiakes DJ, Kleiman NS, et al. Combining antiplatelet and anticoagulant therapies. J Am Coll Cardiol. 2009;54:95-109.

59. Weitz JI. Factor Xa or thrombin: is thrombin a better target? J Thromb Haemost. 2007;5:65-67.

60. Mackman N. Triggers, targets and treatments for thrombosis. Nature. 2008;451:914-918.
Vascular Health and Risk Management

\section{Publish your work in this journal}

Vascular Health and Risk Management is an international, peerreviewed journal of therapeutics and risk management, focusing on concise rapid reporting of clinical studies on the processes involved in the maintenance of vascular health; the monitoring, prevention and treatment of vascular disease and its sequelae; and the involvement of

\section{Dovepress}

metabolic disorders, particularly diabetes. This journal is indexed on PubMed Central and MedLine. The manuscript management system is completely online and includes a very quick and fair peer-review system, which is all easy to use. Visit http://www.dovepress.com/ testimonials.php to read real quotes from published authors. 